\title{
Using capnography: Delivery of an education package for recovery nursing staff.
}

\author{
R. Clarke, E. Birch, A. Clark, R. Junkin \\ Department of Anaesthetics, University Hospital Crosshouse, Kilmarnock, UK
}

\section{Background:}

AAGBI guidelines ${ }^{1}$ advocate the use of capnography for all patients who are deeply sedated or have a supraglottic airway or endotracheal tube in situ. The 4th National Audit Project highlighted the role of capnography in earlier identification of obstructed airways in recovery, noting the limitations associated with poor interpretation ${ }^{2}$. Having identified limited use of capnography in recovery and poor nurse confidence in interpretation, we planned an education program to address this.

\section{Methods and Materials:}

An education package for recovery staff was developed from an already established program ${ }^{3}$. A survey of confidence in capnography use and an assessment of knowledge was undertaken by the staff before and after the education sessions. The education was delivered as small group tutorials covering practical aspects of capnography use. Test scores, maximum 21, were compared using a paired student's t-test. Confidence was reported on a scale of 1 (no confidence) to 5 (very confident).

\section{Results:}

Twenty recovery staff members (83\%) attended tutorials in 5 groups. The mean test score rose from 7.85 $(S D=3.34)$ to $17.45(S D=2.68)$ following the education session $(p<0.05)$, see Fig 1 . The percentage of nurses who rated their confidence as 3 or more rose from $13 \%$ to $100 \%$ following the education package, see Fig. 2.
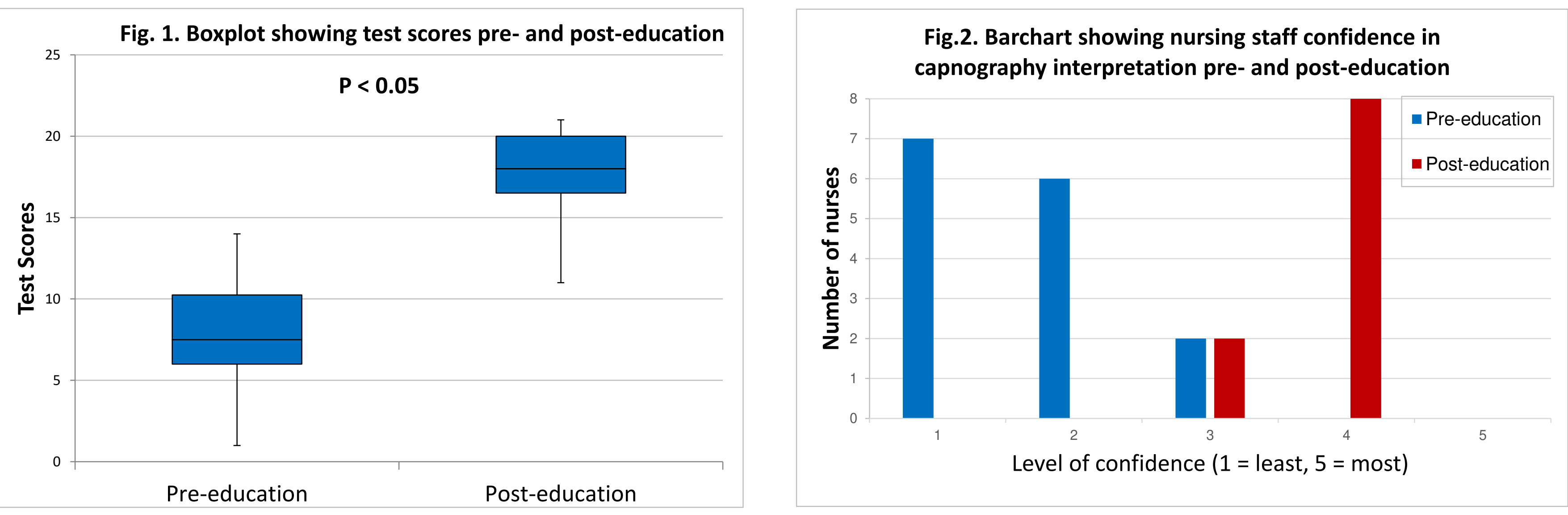

\section{Conclusion:}

The results show the education package has increased recovery staff knowledge and confidence. This improvement has already led to greater adoption of capnography use in recovery. Ultimately this should improve patient safety by aligning our unit with national standards.

\section{References:}

1. Association of Anaesthetists of Great Britain and Ireland. Recommendations for standards of monitoring during anaesthesia and recovery 2015. Anaesthesia 2016; 71: 85-93

2. TM Cook, $N$ Woodall, C Frerk. Major complications of airway management in the UK: Results of the Fourth National Audit Project of the Royal College of Anaesthetists and the Diffcult Airway Society. Part 1: Anaesthesia. British Journal of Anaesthesia 2011; 106(5): 617-631

3. Capnography resources. Scottish Paediatric Anaesthetic Network. http://www.span.scot.nhs.uk/?page id=873 [Accessed 15 May 2018\} 